\title{
Micromachined Vibratory Gyroscopes Controlled by a High-Order Bandpass Sigma-Delta Modulator
}

\author{
Yufeng Dong, Michael Kraft, and William Redman-White, Member, IEEE
}

\begin{abstract}
This work reports on the design of novel closed-loop control systems for the sense mode of a vibratory-rate gyroscope based on a high-order sigma-delta modulator $(\Sigma \Delta M)$. A low-pass and two distinctive bandpass topologies are derived, and their advantages discussed. So far, most closed-loop force-feedback control systems for these sensors were based on low-pass $\Sigma \Delta$ M's. Usually, the sensing element of a vibratory gyroscope is designed with a high quality factor $Q$ to increase the sensitivity and, hence, can be treated as a mechanical resonator. Furthermore, the output characteristic of vibratory rate gyroscopes is narrowband amplitude-modulated signal. Therefore, a bandpass $\Sigma \Delta \mathrm{M}$ is a more appropriate control strategy for a vibratory gyroscope than a low-pass $\Sigma \Delta \mathrm{M}$. Using a high-order bandpass $\Sigma \Delta \mathrm{M}$, the control system can adopt a much lower sampling frequency compared with a low-pass $\Sigma \Delta M$ while achieving a similar noise floor for a given oversampling ratio (OSR). In addition, a control system based on a high-order bandpass $\Sigma \Delta M$ is superior as it not only greatly shapes the quantization noise, but also alleviates tonal behavior, as is often seen in low-order $\Sigma \Delta M$ control systems, and has good immunities to fabrication tolerances and parameter mismatch. These properties are investigated in this study at system level.
\end{abstract}

Index Terms-Bandpass, gyroscope, micromachined, sigmadelta $(\Sigma \Delta)$, vibratory.

\section{INTRODUCTION}

$\mathbf{C}$ LOSED-LOOP control systems using a sigma-delta modulator $(\Sigma \Delta \mathrm{M}$ for inertial sensors are attractive due to the fact that forced rebalanced feedback can increase the bandwidth, linearity, and dynamic range of the sensor, and, most importantly, the closed-loop control system alleviates performance degradations due to microfabrication tolerances. Control systems for micromachined inertial sensors based on a low-pass $\Sigma \Delta \mathrm{M}$ have been used as an effective control strategy for many years [1]. Most reported sensors to date only use the sensing element as a low-pass noise-shaping filter [2]-[4]. Consequently, due to only second-order noise shaping, the quantization noise is the dominating noise source, which limits the overall performance. Recently, high-order low-pass $\Sigma \Delta$ modulators were used for the control system of capacitive inertial sensors [5], [6]. Micromachined vibratory gyroscopes operate on the principle

Manuscript received March 22, 2006; revised July 11, 2006 and July 12, 2006. This is an expanded paper from the Sensors 2005 Conference. The associate editor coordinating the review of this paper and approving it for publication was Prof. P. Regtien.

The authors are with the Nanoscale Systems Integration Group, School of Electronics and Computer Science, University of Southampton, Southampton SO17 1JB, U.K. (e-mail: yd@ecs.soton.ac.uk; mk1@ecs.soton.ac.uk; wrw@ecs.soton.ac.uk).

Color versions of one or more of the figures in this paper are available online at http://ieeexplore.ieee.org.

Digital Object Identifier 10.1109/JSEN.2006.888604
TABLE I

PARAMETERS OF THE GYROSCOPE USED FOR SIMULATIONS

\begin{tabular}{|c|c|}
\hline Variable & Explanations \\
\hline$m=2 \times 10^{-9}(\mathrm{~kg})$ & proof mass \\
\hline$f_{s 1}=2^{23}(\mathrm{~Hz})$ & oversampling frequency (low-pass) \\
\hline$f_{s 2}=2^{16}(\mathrm{~Hz})$ & oversampling frequency (band-pass) \\
\hline$f_{x}=2^{14}(\mathrm{~Hz})$ & $X$-axis drive frequency; $f_{x}=f_{s 2} / 4$ \\
\hline$\omega_{x}=2 \pi f_{x}(\mathrm{rad} / \mathrm{s})$ & $X$-axis resonance frequency (drive) \\
\hline$\omega_{y}=2 \pi f_{y}(\mathrm{rad} / \mathrm{s})$ & Y-axis resonance frequency (sense) \\
\hline$Q_{x}=100$ & quality factor of the drive mode \\
\hline$Q_{y}=100$ & quality factor of the sense mode \\
\hline$X_{0}=2 \times 10^{-6}(\mathrm{~m})$ & amplitude of drive oscillation \\
\hline$\dot{X}_{0}=\omega_{x} \times X_{0}(\mathrm{~m} / \mathrm{s})$ & velocity of drive oscillation \\
\hline$f_{\text {in }}=2^{7}(\mathrm{~Hz})$ & input angular rate signal frequency \\
\hline$O_{S R}=256$ & oversampling ratio \\
\hline$\Omega_{Z}=100(\mathrm{deg} / \mathrm{sec})$ & maximum input angular rate \\
\hline$Y_{G a p}=1(\mu \mathrm{m})$ & gap between sensing capacitors \\
\hline$K_{f b}=3.15 \times 10^{-9}$ & gain of the voltage to force conversion \\
\hline
\end{tabular}

of the transfer of energy between the drive mode and the sense mode of a vibrating mechanical structure caused by Coriolis acceleration; the output signal is amplitude modulated at the drive frequency. To increase the sensitivity, the sensing element usually is designed with a high quality factor $Q$ and, hence, can be treated as a resonator. Therefore, similar principles as for the design of electronic $\Sigma \Delta$ bandpass A/D converters (ADCs) lend themselves to be applied to vibratory gyroscopes. Moreover, the control system using a high-order bandpass $\Sigma \Delta \mathrm{M}$, as proposed in this study, can provide much better noise shaping than when using a second-order $\Sigma \Delta \mathrm{M}$ where the sensing element only constitutes the loop filter in the forward path [7]. The high-order $\Sigma \Delta \mathrm{M}$ interface for a gyroscope was recently described in [5], where the sensing element is cascaded by two electronic integrators to form a fourth-order loop. However, this is a low-pass $\Sigma \Delta \mathrm{M}$ and therefore requires a relatively high sampling frequency, which puts demanding requirements on the circuit implementation. As a general guideline for the design of electromechanical $\Sigma \Delta \mathrm{M}$ 's, it is desirable to implement a control loop with comparable noise levels among mechanical (Brownian) noise, electronic noise, and quantization noise. Based on a linearized model for the quantizer [8], the signal transfer function $(S T F)$, quantization noise transfer function $(Q N T F)$, and electronic noise transfer function $(E N T F)$ will be compared with a low-pass fifth-order $\Sigma \Delta \mathrm{M}$ control loop and two distinct topologies of bandpass $\Sigma \Delta \mathrm{M}$ control loops. Additionally, extensive system level simulations in Simulink/Matlab are used to investigate their noise shaping abilities [9]. 


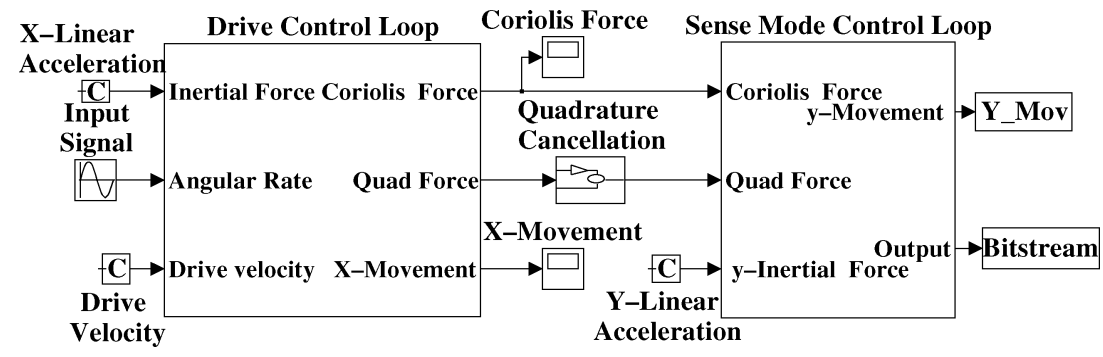

Fig. 1. Vibratory gyroscope diagram consisting of the drive mode and sense mode control loops.

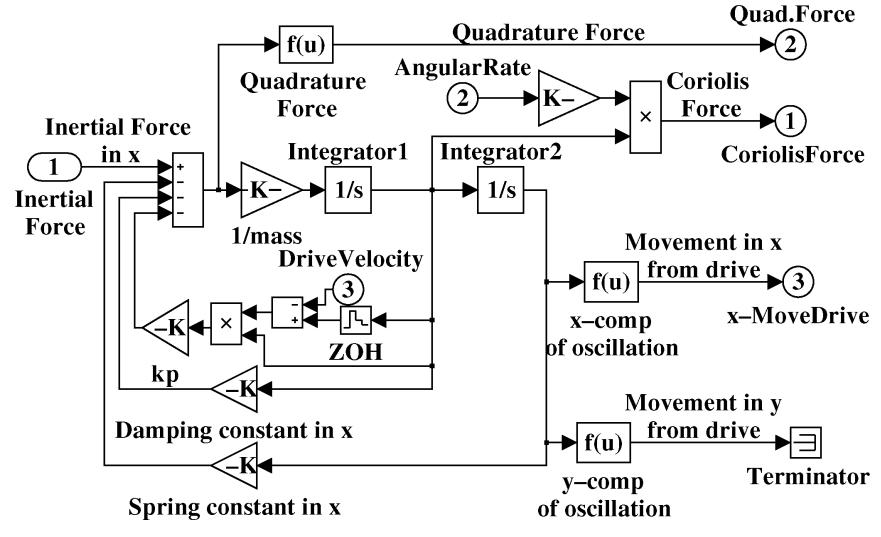

Fig. 2. Drive-mode control-loop block of Fig. 1.

\section{System-LeVel Model of a Vibratory Gyroscope}

As the sensing element, a typical micromachined gyroscope packaged at ambient atmospheric pressure is assumed for system simulations. Table I lists the parameters of the micromachined gyroscope used for simulation throughout this paper. The continuous-time transfer function of the sensing element in the sense direction is given by

$$
M_{m}(s)=\frac{1}{m s^{2}+b s+k}=\frac{1 / m}{s^{2}+\frac{\omega_{y}}{Q_{y}} s+\omega_{y}^{2}}
$$

where $m$ is the mass of the sensing element, $b$ is the damping coefficient, $k$ is the spring stiffness, $\omega_{y}=\sqrt{k / m}$ is the resonant frequency, and $Q_{y}=\sqrt{m k} / b$ is the quality factor of the sense mode. The following analysis uses its equivalent discrete-time transfer function $M_{m}(z)$ in the $z$-domain, which can be obtained using an impulse-invariant transformation [7].

Fig. 1 shows a block diagram of a closed-loop vibratory gyroscope, which consists of the drive mode and sense mode control loops. The drive mode control loop is shown in Fig. 2, and its main function is to precisely keep the drive oscillation at constant frequency $\left(\omega_{x}\right)$ and amplitude $\left(X_{o}\right)$. The motion of the vibrating proof mass is described by $x=X_{0} \sin \left(\omega_{x} t\right)$. If the sensor is rotated around the $z$-axis with an angular rate $\Omega_{z}$, the Coriolis force resulting along the $y$-direction on the proof mass is given by

$$
F_{\text {Coriolis }}=2 m \frac{d x}{d t} \Omega_{z}=2 m X_{0} \omega_{x} \Omega_{z} \cos \left(\omega_{x} t\right) .
$$

The focus of the following discussion, however, is the sensemode control system which consists of an electromechanical
$\Sigma \Delta \mathrm{M}$. There are several possible choices for the sense control loop: 1) it can be either a low-pass or a bandpass $\Sigma \Delta \mathrm{M}$ and 2 ) it can be either a second-order (sensing element only as loop filter) or a high-order $\Sigma \Delta \mathrm{M}$. Any combination of these choices is also possible. In the case of a high-order, low-pass $\Sigma \Delta \mathrm{M}$, the sensing element is cascaded by electronic integrators, whereas in the case of a high-order bandpass $\Sigma \Delta \mathrm{M}$, the sensing element is cascaded with electronic resonators.

\section{HIGH-ORDER LOW-PASS $\Sigma \Delta \mathrm{M}$ FOR THE SENSE-MODE CONTROL SYSTEM}

As a first topology, a fifth-order low-pass $\Sigma \Delta \mathrm{M}$ with multifeedback and local resonators (MFLRs) [10] is investigated as a closed-loop control system for the sense mode of a vibratory gyroscope. The design is shown in Fig. 3 as a Simulink model.

The sensing element $M_{m}(s)$ is used as the first integrator. The gain $K_{p o}$ of the preamplifier represents the conversion gain from the proof mass displacement to voltage. $K_{\mathrm{bst}}$ is the gain of an additional boost amplifier. A lead phase compensator $C_{p}(z)=\left(1-0.9 z^{-1}\right)$ is located after the pickoff interface. Three electronic integrators $H_{1}(z), H_{2}(z)$, and $H_{3}(z)$ have a transfer function of $H_{i}(z)=\left(z^{-1} / 1-z^{-1}\right)(i=1,2,3)$. $a_{1}, a_{2}$, and $a_{3}$ are gain coefficients used for integrator output scaling. The two local resonators are formed by gain constants $g_{1}$ and $g_{2}$, which create a notch in the signal band to further suppress the quantization noise. The local feedback paths create complex pairs of zeros in the QNTF, which produce a notch [8] to cause faster decay in the stop-band and thus further suppress the quantization noise presented here. Unlike a second-order electromechanical $\Sigma \Delta \mathrm{M}$, a high-order single-loop electromechanical $\Sigma \Delta \mathrm{M}$ may apply more local feedback paths in the loop filter to shape the end-of-the-band quantization noise. All values of the gain constants are given in the figure. The topology is derived by applying a design methodology based on optimization and stability criteria which is described in more detail in [11]. In most devices, the electronic noise from circuits is the dominant noise source, therefore an input-referred electronic white noise source with a typical value for the power spectral density of $6 \mathrm{nV} / \sqrt{\mathrm{Hz}}$ is placed at the input of the pickoff amplifier.

An electrostatic feedback force is generated on the proof mass, by applying a voltage to the electrodes of the sensing element. For a given sensing element in a closed-loop control system, the dynamic range is determined by the electrostatic force which can be set by the feedback voltage $V_{\mathrm{fb}} . K_{\mathrm{fb}}$ is the 


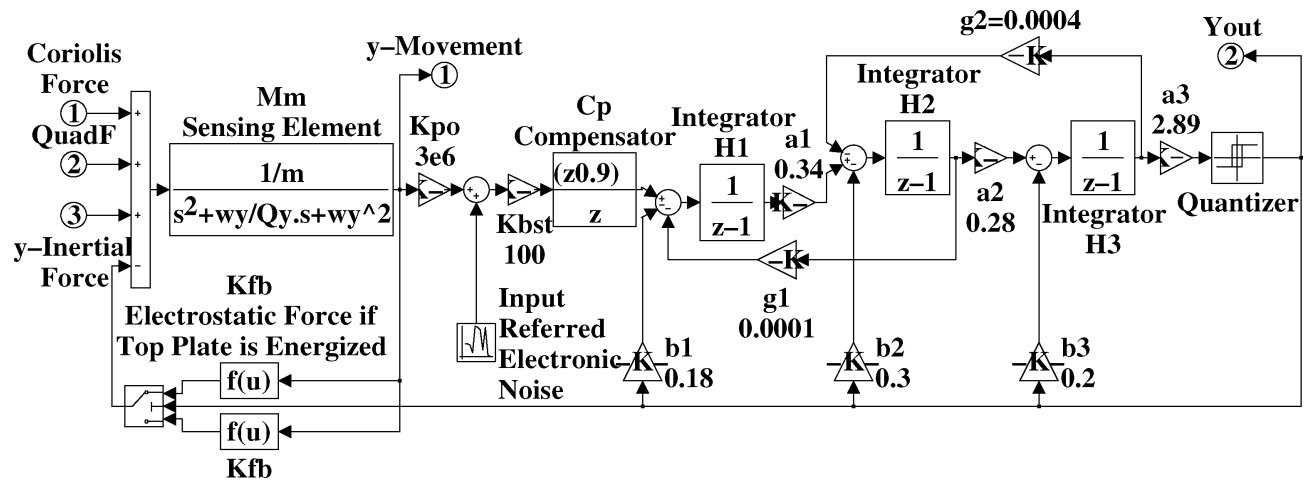

Electrostatic Force if

Bottom Plate is Energized

Fig. 3. Sense-mode control loop based on a fifth-order low-pass $\Sigma \Delta M$ with MFLRs.

gain defined by the voltage to electrostatic force conversion on the proof mass in the feedback path and given by

$$
K_{\mathrm{fb}}=\operatorname{sgn}\left(Y_{\text {out }}\right) \frac{\varepsilon_{0} A_{\mathrm{fb}} V_{\mathrm{fb}}^{2}}{2\left(d_{0}+\operatorname{sgn}\left(Y_{\text {out }}\right) \cdot x\right)^{2}}
$$

where $\varepsilon_{0}$ is the dielectric constant, $A_{f b}$ is the area of the feedback electrode, $d_{0}$ is the nominal gap between proof mass and the electrodes to either side, $x$ is the proof mass deflection from its rest position, and $Y_{\text {out }}$ is the quantizer output bitstream is normalized to be $\pm 1 \mathrm{~V}$.

A sampling frequency $f_{s 1}=2^{23} \mathrm{~Hz}(\approx 8 \mathrm{MHz})$ is chosen for the control system as shown in Fig. 3, which results in an oversampling ratio (OSR $=f_{s 1} /\left(2 f_{y}\right)$ ) of 256 . The transfer functions $S T F, E N T F$, and $Q N T F$ are given by [12]

$$
\begin{aligned}
\operatorname{STF}_{1}(z) & =\frac{\kappa_{m}(z) K_{q} \prod_{i=1}^{3} H_{i}(z) a_{i} / K_{f b}}{L_{1}(z)} \\
\operatorname{ENTF}_{1}(z) & =\frac{\kappa_{m}(z) K_{q} \prod_{i=1}^{3} H_{i}(z) a_{i} /\left(K_{f b} M_{m}(z) K_{p o}\right)}{L_{1}(z)} \\
\operatorname{NNTF}_{1}(z) & =\frac{1+\sum_{i=1}^{2} H_{i}(z) a_{i} H_{i+1}(z) g_{i}}{L_{1}(z)}
\end{aligned}
$$

where

$$
\begin{aligned}
L_{1}(z) & =1+\kappa_{m}(z) \prod_{i=1}^{3} H_{i}(z) a_{i}+K_{q} \sum_{i=1}^{3} b_{i} \prod_{j=i}^{3} H_{j}(z) a_{j} \\
& +\sum_{i=1}^{2} a_{i} g_{i} \prod_{j=i}^{i+1} H_{j}(z)+H_{1}(z) a_{1} H_{2}(z) g_{1} a_{3} H_{3}(z) b_{3} K_{q}
\end{aligned}
$$

and $\kappa_{m}(z)=K_{f b} M_{m}(z) K_{p o} K_{b s t} C_{p}(z)$.

The notch frequency is provided by the local resonators [the numerator of (6)] and given by

$$
\omega=\sqrt{\omega_{1} \omega_{2} g_{1} a_{1}+\omega_{2} \omega_{3} g_{2} a_{2}}
$$

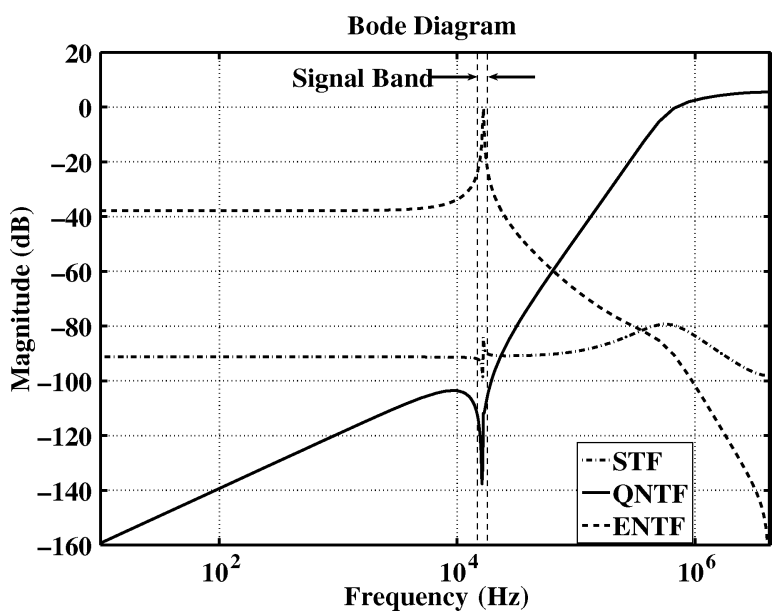

Fig. 4. Bode diagram of $S T F, Q N T F$, and $E N T F$ of the sense mode control system based on a fifth-order low-pass $\Sigma \Delta \mathrm{M}$ depicted in Fig. 3.

where $\omega_{1}, \omega_{2}$, and $\omega_{3}$ are the unity-gain frequencies of the first, second, and third integrators, respectively (usually the unitygain frequencies of integrators are chosen as the sampling frequency $f_{s}$ ). Because the notch is created in the band of interest, the local feedback gains $\left(g_{1}\right.$ and $\left.g_{2}\right)$ are very small and have no effect on the loop stability. The notch frequency can be similarly analyzed for the local resonators of the high-order bandpass electromechanical $\Sigma \Delta$ M's which will be discussed in Section V.

The Bode diagram of the transfer functions (STF, QNTF, and $E N T F$ ) is shown in Fig. 4. It can be seen that the $S T F$ has a sharp peak around the drive resonant frequency $f_{x}$ due to the quality factor of 100 , and therefore the signal bandwidth is very narrow, and there is no flat region in the signal band. The output bitstream spectrum is shown in Fig. 5(a) with a close-up view around the signal bandwidth [see Fig. 5(b)], which indicates that the signal is symmetrically located at $f_{y} \pm f_{\text {in }}$. The signal-to-noise ratio (SNR) is calculated to be about $82 \mathrm{~dB}$ in a $256-\mathrm{Hz}$ bandwidth. It should be noted that the two signal peaks have 6-dB difference, which is due to the signal has a sharp transfer function in the signal band. This further verified the $S T F$ Bode diagram shown in Fig. 4. Bandwidth is a critical 


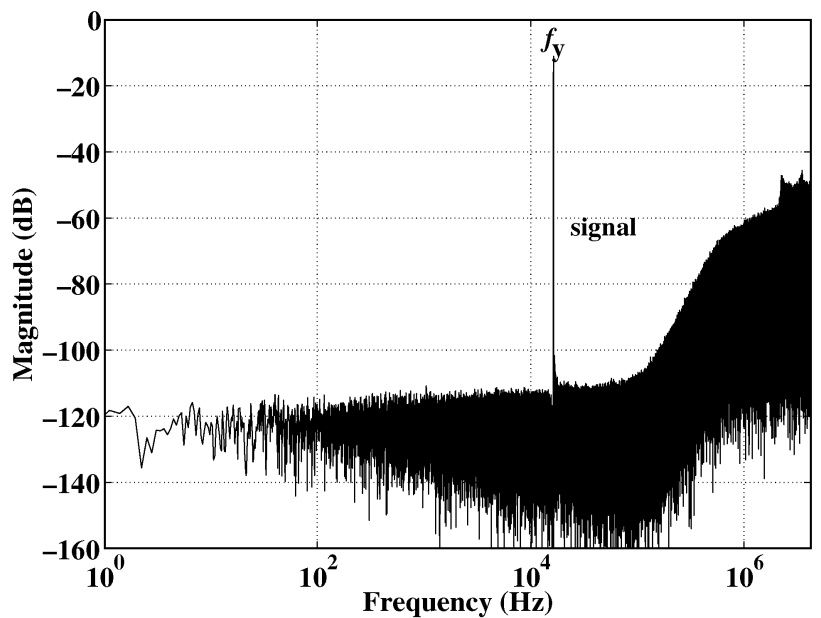

(a)

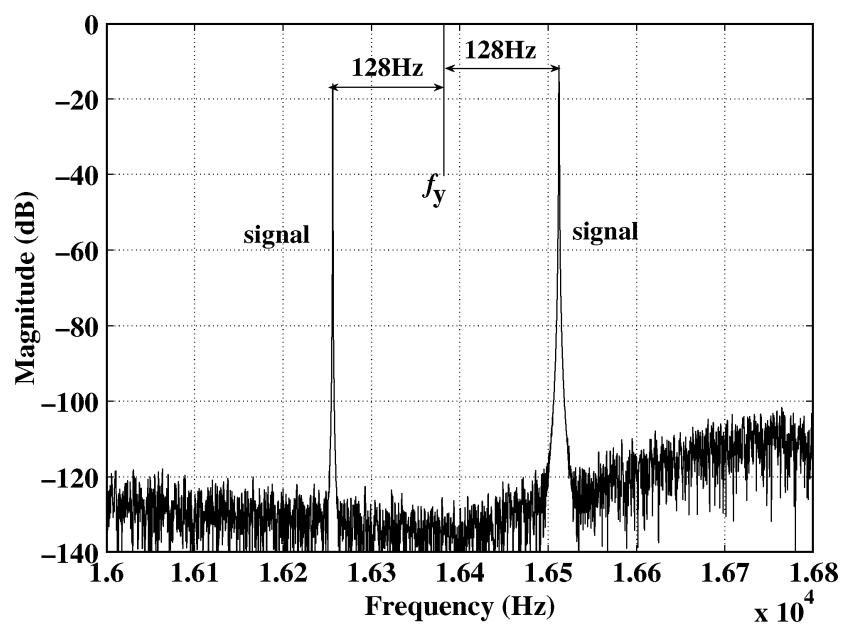

(b)

Fig. 5. Output bitstream spectrum of the control system based on a fifth-order low-pass $\Sigma \Delta \mathrm{M}$ depicted in Fig. 3. (a) Full view of the output bitstream spectrum. (b) Close-up view of the signal band of (a).

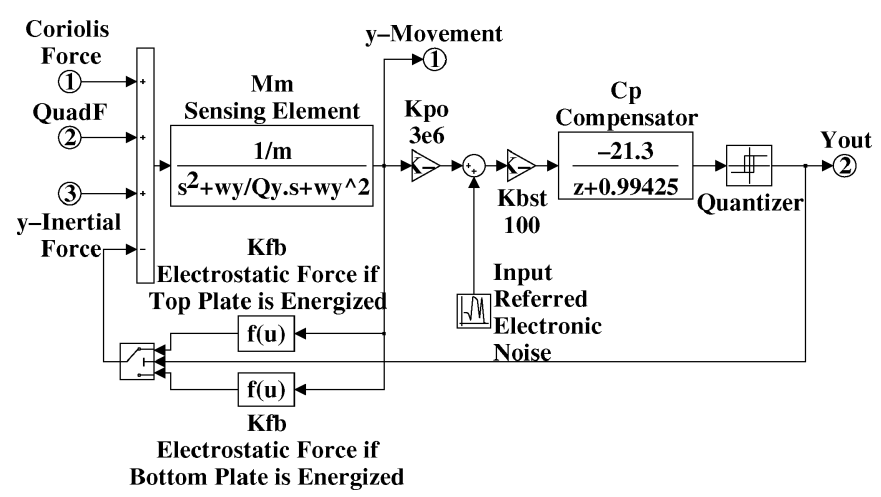

Fig. 6. Sense-mode control loop based on a second-order bandpass $\Sigma \Delta M$.

factor for the dynamic performance of a micromachined vibratory gyroscope [13]. Thus, using a control system based on a low-pass $\Sigma \Delta \mathrm{M}$ restricts vibratory rate gyroscopes to relatively narrow-band applications.

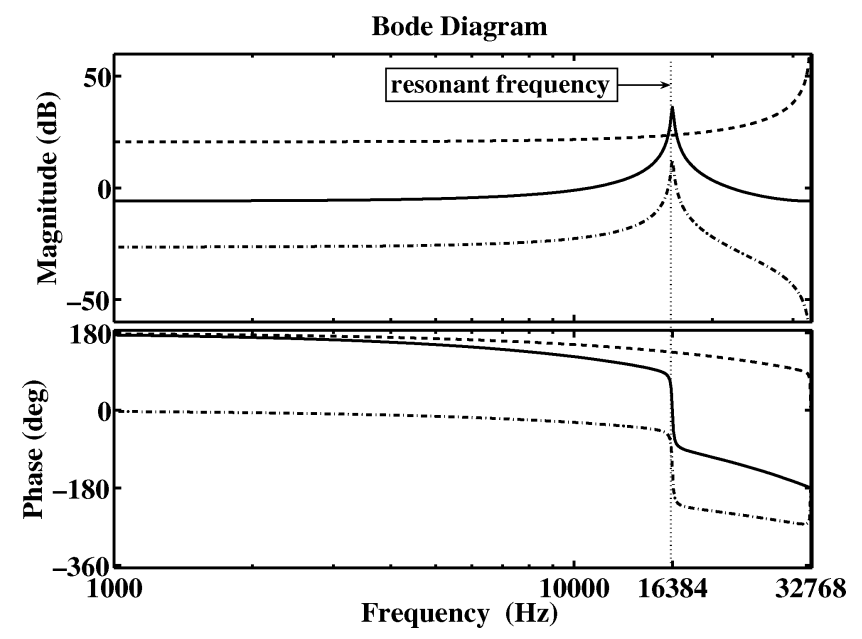

Fig. 7. Bode diagram of the compensator, sensing element, and the compensated sensing element for the sense-mode control system using a bandpass $\Sigma \Delta \mathrm{M}$.

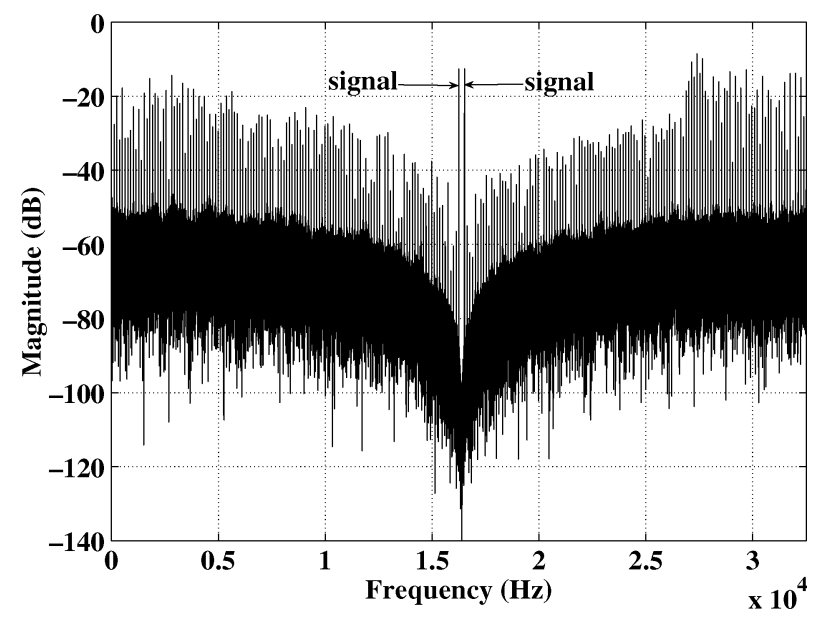

Fig. 8. Output bitstream spectrum of the control system using a second-order bandpass $\Sigma \Delta \mathrm{M}$, showing significant tonal behavior.

\section{SECOND-ORDER BANDPASS $\Sigma \Delta \mathrm{M}$ FOR THE SENSE-MODE CONTROL SYSTEM}

The sensing element of vibratory rate gyroscope is equivalent to a resonator in the electrical domain, therefore it lends itself to be incorporated in a bandpass $\Sigma \Delta \mathrm{M}$ control loop. As a first approach, a bandpass $\Sigma \Delta \mathrm{M}$ loop is designed that uses the mechanical resonator only as the loop filter. This results in a sense-mode control system having a topology of a second-order bandpass $\Sigma \Delta \mathrm{M}$, which is shown in Fig. 7.

The difference in a second-order low-pass $\Sigma \Delta \mathrm{M}$ (as described in [7]) is that the sampling frequency can be as low as four times the resonant frequency $\left(f_{s}=4 f_{y}\right)$. Furthermore, the compensator has a different format compared with the compensator for a low-pass $\Sigma \Delta \mathrm{M}$ interface. It compensates for the phase lag of the sensing element at high frequencies to provide sufficient loop phase margin for stable operation. The design of such a compensator using classic control theory [14] is also a first step toward the design of a high-order bandpass loop. The compensator used here is given by

$$
C_{p}(z)=\frac{-22.13}{z+0.9942} \text {. }
$$




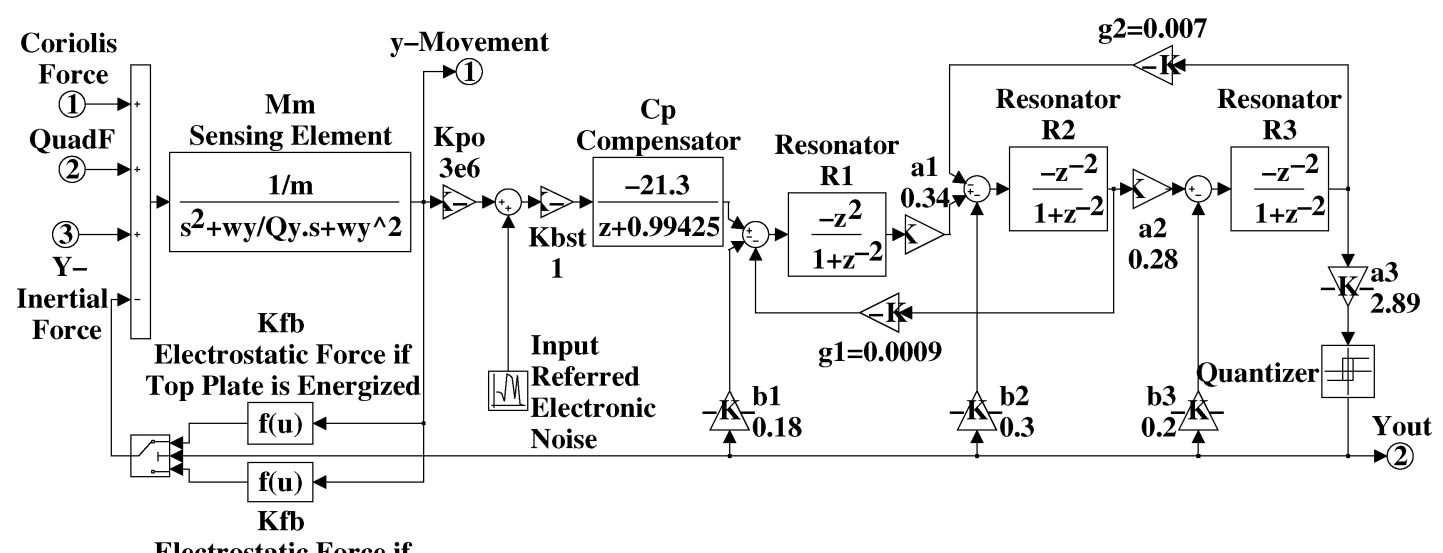

Electrostatic Force if

Bottom Plate is Energized

Fig. 9. Sense-mode control system based on an eighth-order bandpass $\Sigma \Delta M$ with MFLRs.

The Bode diagram of the compensator and sensing element is plotted in Fig. 7. With the compensator, the phase lag originating from the sensing element is sufficiently compensated at high frequencies and provides $90^{\circ}$ phase lead at $f_{s} / 2$ compared with the case without compensation. The output bitstream spectrum is shown in Fig. 8. If distortion is not included, the SNR is about $63 \mathrm{~dB}$ in a bandwidth of $256 \mathrm{~Hz}$. However, there are many idle tones in the spectrum, which is due to the quantization noise no longer being white. Calculating the SNR including harmonic distortion results in a value of about $35 \mathrm{~dB}$, which is unacceptably low for most applications. For a lower order $\Sigma \Delta \mathrm{M}$, the additive white noise approximation of the nonlinear one-bit quantizer is not a valid assumption. These tones may fold into the signal band and degrade the SNR. In contrast, a high-order $\Sigma \Delta \mathrm{M}$ suffers less tonal behavior [8]. Therefore, the control system using a high-order $\Sigma \Delta \mathrm{M}$ can help to reduce these tones. This is verified in the subsequent sections.

\section{HIGH-ORDER BANDPASS $\Sigma \Delta \mathrm{M}$ FOR THE SENSE-MOdE CONTROL SYSTEM}

\section{A. Low-Pass to Bandpass Transformation}

The design methodology of a high-order bandpass $\Sigma \Delta \mathrm{M}$ is based on the application of a low-pass to bandpass transformation on a more commonly used low-pass filter topology. One of standard transformations [8] is

$$
z \rightarrow-z^{2}
$$

A low-pass $\Sigma \Delta \mathrm{M}$ of order $N$ can be converted to a bandpass modulator of order $2 N$ with a center frequency of $f_{0}=f_{s} / 4$ ( $f_{s}$ is the sampling frequency); this preserves both the stability characteristics and the noise-shaping properties of the original modulator [8]. The low-pass $\Sigma \Delta \mathrm{M}$ has $Q N T F$ zeros at or near $z=1$ (which corresponds to dc), whereas the bandpass $\Sigma \Delta \mathrm{M}$ has $Q N T F$ zeros at or near $z= \pm j$ (which corresponds to $f_{0}=$ $\left.f_{s} / 4\right)$. In a bandpass $\Sigma \Delta \mathrm{M}$, the equivalent of the integrators in the low-pass $\Sigma \Delta \mathrm{M}$ is a resonator which has a discrete-time transfer function in the format of

$$
R(z)=\frac{-z^{-2}}{1+z^{-2}} .
$$

The transformation of (10) is only applied to the electronic integrators, but not to the sensing element as it is a mechanical resonator.

\section{B. High-Order Bandpass $\Sigma \Delta \mathrm{M}$ With MFLRs}

Applying this transformation to the fifth-order low-pass $\Sigma \Delta \mathrm{M}$ described above (Fig. 3) results in an eighth-order bandpass $\Sigma \Delta \mathrm{M}$, which is shown in Fig. 9. The control loop consists of the same micromachined sensing element $M_{m}(s)$ (i.e., the first resonator) cascaded with pickoff amplifiers, a lead phase compensator $C_{p}(z)$, and three electronic resonators $R_{1}(z)$, $R_{2}(z)$, and $R_{3}(z) . a_{1}, a_{2}$, and $a_{3}$ are gain constants used for scaling the resonator output amplitude. The gain constants $g_{1}$ and $g_{2}$ in the feedback path are used to generate two local resonators. Thus, a notch is created in the signal band to further suppress the quantization noise. The OSR $\left(=f_{s 2} / 2 f_{\text {in }}\right)$ for a bandpass $\Sigma \Delta \mathrm{M}$ is the ratio between the sampling frequency $f_{s 2}$ and twice the input signal frequency $f_{\text {in }}$; therefore, to obtain the same value of the OSR as for the low-pass $\Sigma \Delta \mathrm{M}$, the sampling frequency is now reduced to $f_{s 2}=2^{16} \mathrm{~Hz}(\approx 63 \mathrm{kHz})$. The transfer functions $S T F, E N T F$, and $Q N T F$ are given by

$$
\begin{aligned}
\operatorname{STF}_{2}(z) & =\frac{\kappa_{m}(z) K_{q} \prod_{i=1}^{3} R_{i}(z) a_{i} / K_{f b}}{L_{2}(z)} \\
\operatorname{ENTF}_{2}(z) & =\frac{\kappa_{m}(z) K_{q} \prod_{i=1}^{3} R_{i}(z) a_{i} /\left(K_{f b} M_{m}(z) K_{p o}\right)}{L_{2}(z)} \\
\operatorname{QNTF}_{2}(z) & =\frac{1+\sum_{i=1}^{2} R_{i}(z) a_{i} R_{i+1}(z) g_{i}}{L_{2}(z)}
\end{aligned}
$$

where

$$
\begin{aligned}
L_{2}(z) & =1+\kappa_{m}(z) K_{q} \prod_{i=1}^{3} R_{i}(z) a_{i}+K_{q} \sum_{i=1}^{3} b_{i} \prod_{j=i}^{3} R_{j}(z) a_{j} \\
& +\sum_{i=1}^{2} a_{i} g_{i} \prod_{j=i}^{i+1} R_{j}(z)+R_{1}(z) a_{1} R_{2}(z) g_{1} a_{3} R_{3}(z) b_{3} K_{q} .
\end{aligned}
$$

The Bode diagram of these transfer functions is shown in Fig. 10. A high-order $\Sigma \Delta \mathrm{M}$ is a conditionally stable closed-loop 


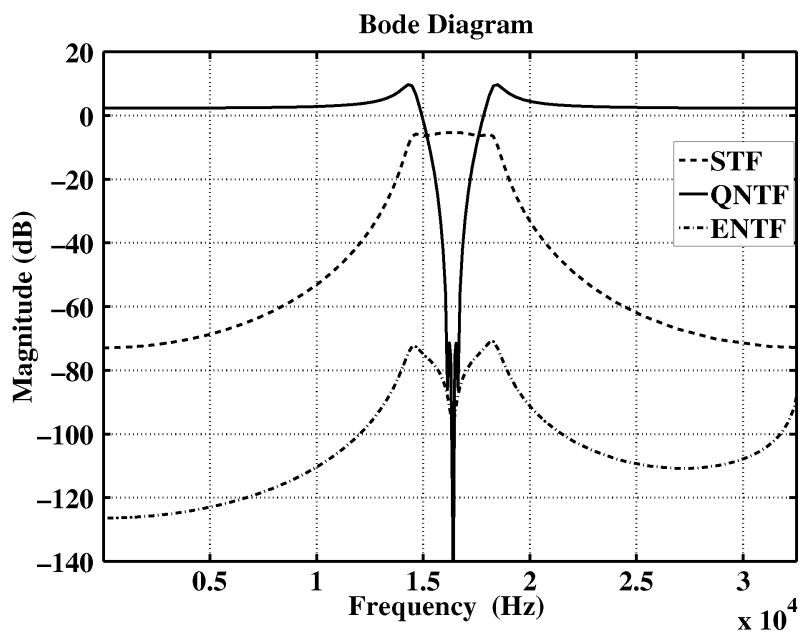

Fig. 10. Bode diagram of the $S T F, Q N T F$, and $E N T F$ of the eighth-order bandpass $\Sigma \Delta M$ with MFLRs (shown in Fig. 9).

system [8]. The open-loop filter $H(z)=1 / Q N T F(z)-1$ determines the loop stability. According to classic control theory [14], a stable system must have all roots within the unit circle. The root locus of the open-loop filter is plotted in Fig. 11(a), with a close-up shown in Fig. 11(b). In order to ensure that the poles lie within the unit circle, the critical minimum gain of the quantizer is $K_{q \min }=0.693$. It reveals that one pole pair moves out of the unit circle with decreasing quantizer gain $K_{q}$ and finally results in an unstable operation. This could be caused by an input signal overload, therefore instability detection and saturation recovery techniques should be applied to a hardware implementation of a high-order $\Sigma \Delta \mathrm{M}$ [15]; however, this was not considered in this work. For a full-scale input signal, the spectrum of the output bitstream is shown in Fig. 12. The signal is symmetrically located at $f_{x} \pm f_{\text {in }}$. Tones only appear at low or high frequencies, but not in the vicinity of the signal band. The SNR is calculated to be $93 \mathrm{~dB}$ in a $256-\mathrm{Hz}$ signal bandwidth.

\section{High-Order Bandpass $\Sigma \Delta \mathrm{M}$ Using Feedforward Loops With a Local Resonator (FFLR)}

Fig. 13 shows an eighth-order bandpass $\Sigma \Delta \mathrm{M}$ topology employing FFLR. The advantage compared with the topology presented in the previous section is that the output levels of the electronic resonators is much lower, therefore making this topology more suitable for low-power applications [16]. The control systems consists of the micromachined sensing element $M_{m}(z)$ cascaded with a lead phase compensator $C_{p}(z)$ and three electronic resonators $R_{1}(z), R_{2}(z)$, and $R_{3}(z) . b_{1}, b_{2}$, and $b_{3}$ are gain constants used for scaling the resonator output amplitude. The feedforward loops are formed by the gain constants $a_{1}$, $a_{2}$, and $a_{3}$; the local resonator by the feedback path with gain constant $g$. Thus, a notch is created in the signal band to further suppress the quantization noise. With a sampling frequency $f_{s 2}=2^{16} \mathrm{~Hz}(\approx 63 \mathrm{kHz})$, the OSR $\left(f_{s 2} / 2 f_{\text {in }}\right)$ is equivalent to the low-pass $\Sigma \Delta \mathrm{M}$ shown in Fig. 3. The transfer functions $S T F, E N T F$, and $Q N T F$ are given by

$$
S T F_{3}(z)=\frac{\kappa_{m}(z) K_{q} T(z) / K_{f b}}{L_{3}(z)}
$$

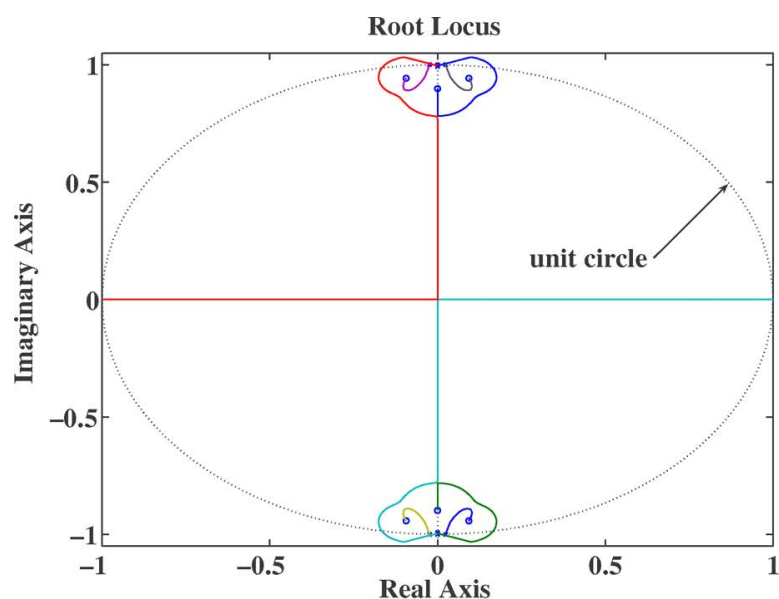

(a)

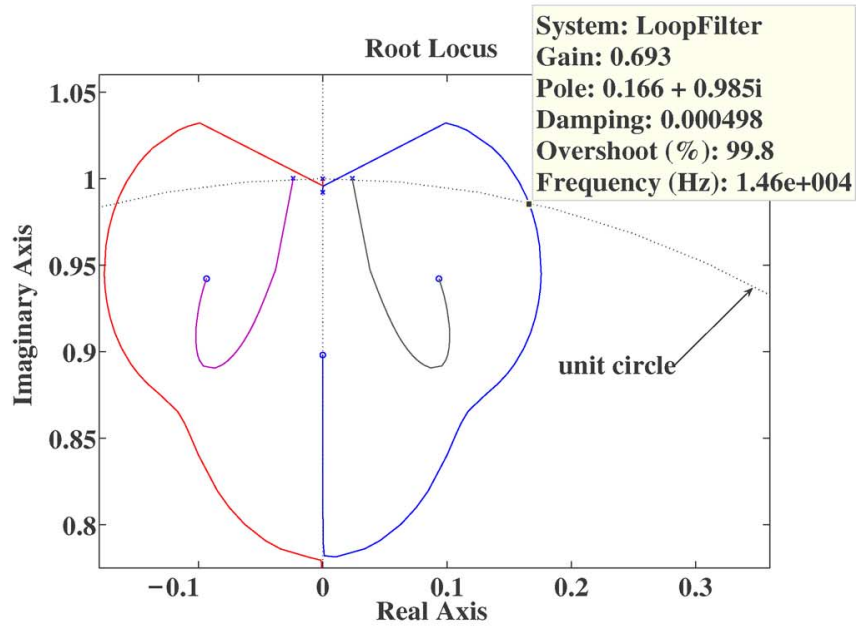

(b)

Fig. 11. Root locus of an open-loop filter of the eighth-order bandpass $\Sigma \Delta \mathrm{M}$ with MFLRs (shown in Fig. 9). (a) Full view of the root locus of open-loop filter. (b) Close-up view of the upper section of (a).

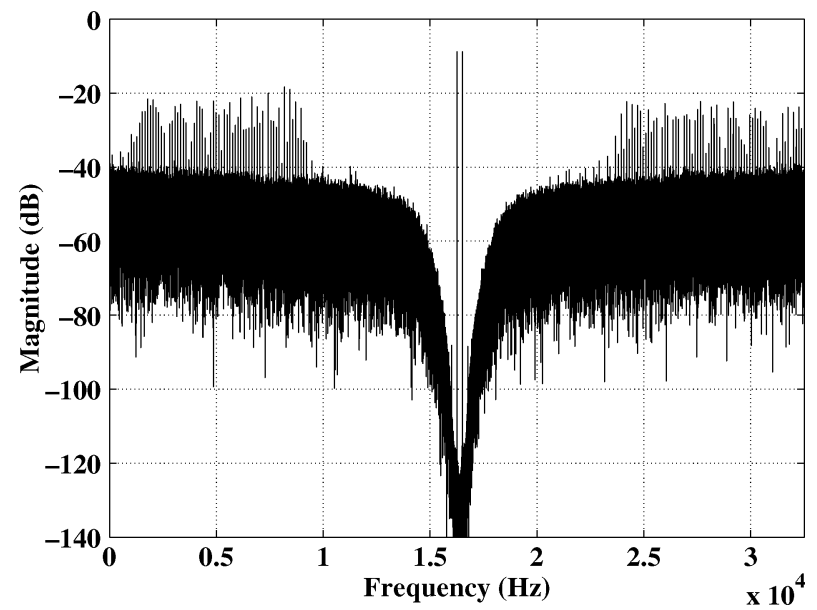

Fig. 12. Output bitstream spectrum of the eighth-order bandpass $\Sigma \Delta \mathrm{M}$ with MFLRs (shown in Fig. 9).

$$
\begin{aligned}
& \operatorname{ENTF}_{3}(z)=\frac{\kappa_{m}(z) K_{q} T(z) / K_{f b} M_{m} K_{p o}}{L_{3}(z)} \\
& \operatorname{NNTF}_{3}(z)=\frac{1+g R_{2}(z) b_{3} R_{3}(z)}{L_{3}(z)}
\end{aligned}
$$




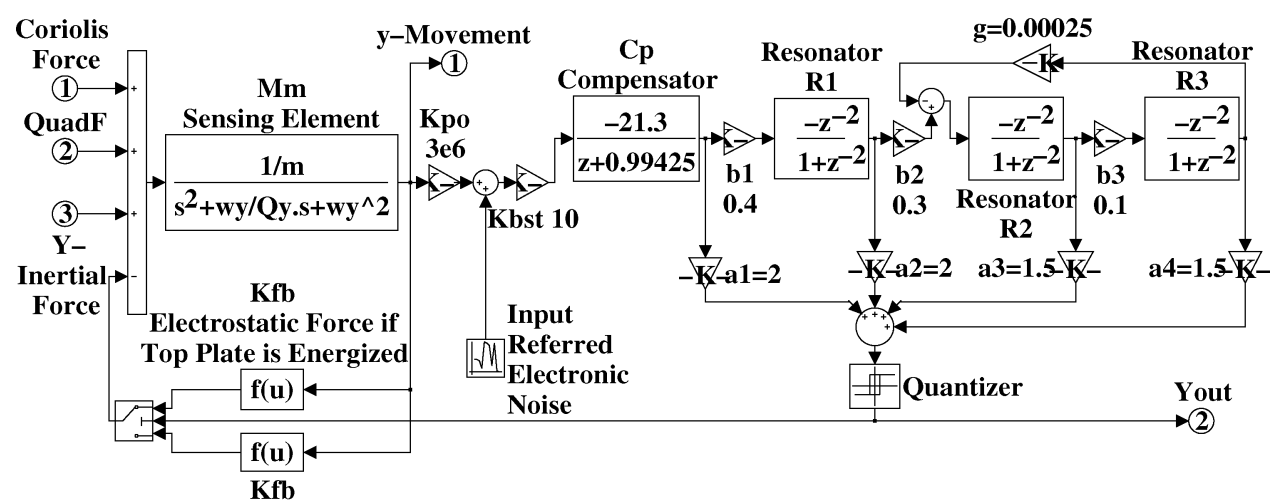

Electrostatic Force if

Bottom Plate is Energized

Fig. 13. Sense-mode control system based on an eighth-order bandpass $\Sigma \Delta M$ with FFLRs.

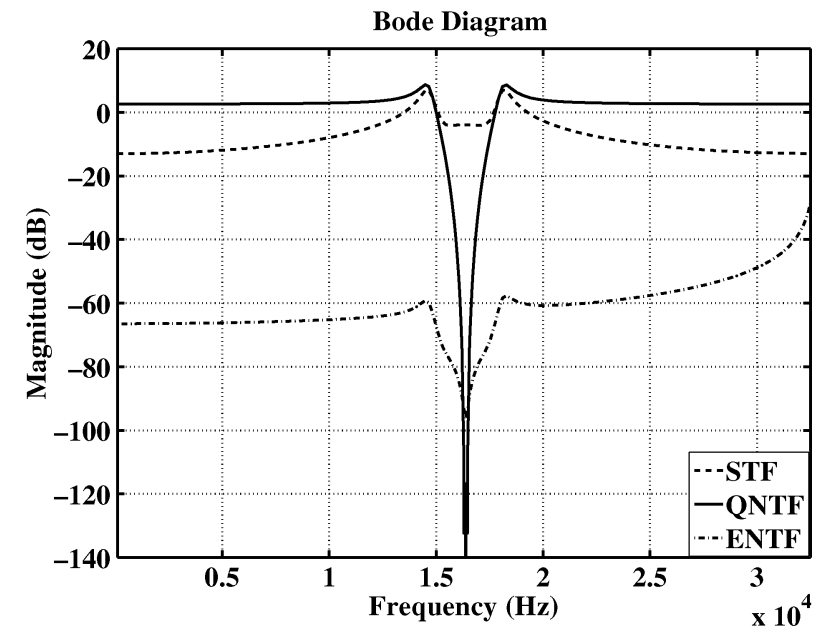

Fig. 14. Bode diagram of the $S T F, Q N T F$, and $E N T F$ of the eighth-order bandpass $\Sigma \Delta M$ with FFLRs (shown in Fig. 13).

where

$$
\begin{aligned}
& L_{3}(z)=1+\kappa_{m}(z) K_{q} \sum_{i=1}^{4} a_{i} \prod_{j=1, j>0}^{i-1} b_{j} R_{j}(z) \\
& +g R_{2}(z) b_{3} R_{3}(z)\left[1-\left(\kappa_{m}(z) K_{q}\left(a_{1}+b_{1} R_{1}(z) a_{2}\right)\right]\right. \\
& T(z)=b_{1} R_{1}(z) b_{2} R_{2}(z)\left(b_{3} R_{3}(z) a_{4}+a_{3}\right) \\
& +\left(1+g R_{2}(z) b_{3} R_{3}(z)\right)\left(b_{1} R_{1}(z) a_{2}+a_{1}\right) .
\end{aligned}
$$

The Bode diagram of these loop transfer functions is shown in Fig. 14. The root locus of the open-loop filter is plotted in Fig. 15(a), with a close-up view shown in Fig. 15(b). In order to ensure that the root locus lies within the unit circle, the critically minimum gain of the quantizer is $K_{q \min }=0.699$. The spectrum of the output bitstream is shown in Fig. 16. The SNR is almost the same as the control system using a multifeedback

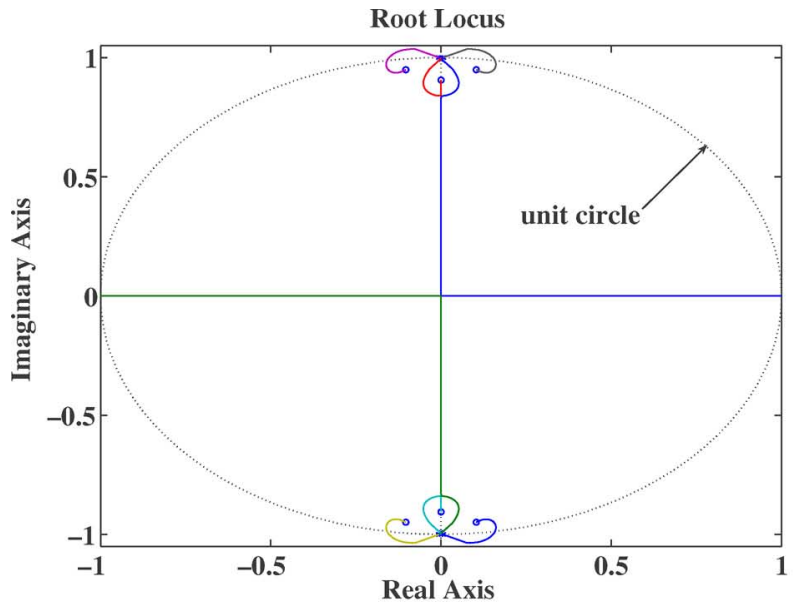

(a)

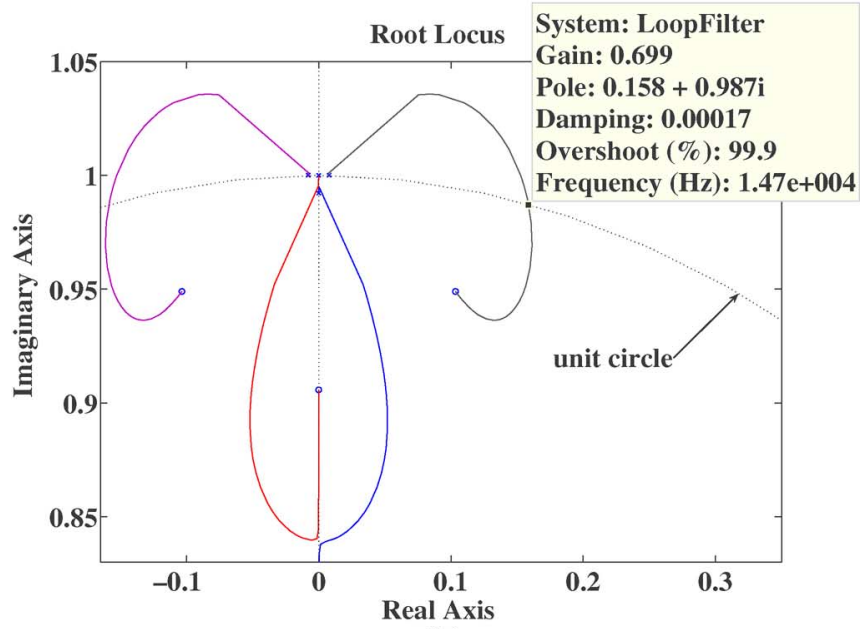

(b)

Fig. 15. Root locus of the open-loop filter of the eighth-order bandpass $\Sigma \Delta \mathrm{M}$ with FFLRs (shown in Fig. 13). (a) Full view of the root locus of the open loop filter. (b) Close-up view of the upper section of (a).

loop topology. The tones are also located at either low or high frequencies having no effect on the signal. 


\section{DISCUSSIONS}

\section{A. Properties of Signal and Noise Transfer Functions}

For the signal transfer function $S T F$ in the two presented high-order bandpass $\Sigma \Delta \mathrm{M}$ 's, the response has a relatively wide $(>1 \mathrm{kHz})$ flat all-pass characteristic in the frequency range of interest. This is in contrast to a narrow signal band in a low-pass $\Sigma \Delta \mathrm{M}$. Therefore, a vibratory-rate gyroscope employing a bandpass $\Sigma \Delta \mathrm{M}$ intrinsically has a much higher signal bandwidth. Another property is that the discrete time circuit blocks are all located after the sensing element, therefore the continuous-time sensing element provides an implicit second-order antialias filtering. This is an advantageous property of all discussed $\Sigma \Delta \mathrm{Ms}$ control systems.

For all high-order topologies presented in this paper, the ratio between the ENTF and the $S T F$ is given by

$$
\frac{\operatorname{ENTF}_{i}(z)}{\operatorname{STF}_{i}(z)}=\frac{1}{M_{m}(z) K_{p o}} \quad(i=1,2,3) .
$$

This is calculated by dividing (4) by (5), (11) by (12), and (14) by (15), respectively. Since the $S T F$ has an all-pass characteristic for the two bandpass topologies, (17) denotes that the electronic noise is shaped by the inverse transfer function of the sensing element. For a micromachined vibratory gyroscope, the $Q$ is relatively high in order to increase its sensitivity. This leads to a better electronic noise shaping in the signal band for a bandpass $\Sigma \Delta \mathrm{M}$ provided that the quality of the sensing element is sufficiently high. The SNR of the two high-order bandpass $\Sigma \Delta$ M's are almost the same: about $93 \mathrm{~dB}$ in a $256-\mathrm{Hz}$ bandwidth, compared with about $82 \mathrm{~dB}$ for the fifth-order, low-pass $\Sigma \Delta \mathrm{M}$. It can be seen from the Bode diagrams and the output bitstream spectra that the noise contribution from the electronic noise is dominant compared with the quantization noise. Therefore, quantization noise is no longer the limiting factor for the sensor performance. To achieve a comparable noise floor in a control system using a low-pass $\Sigma \Delta \mathrm{M}$ and a bandpass $\Sigma \Delta \mathrm{M}$, the sampling frequency of the former has to be much higher (256 times) than that of the latter. This implies a much higher operating frequency of the interface and control circuits for a low-pass $\Sigma \Delta \mathrm{M}$; this is a distinct disadvantage.

\section{B. Quadrature Force Effect on the System Performance}

Thus far, quadrature error was not taken into account. Considering a quadrature force due to microfabrication tolerances assuming a misalignment angle of $\eta=0.001^{\circ}$, the output bitstream spectrum of the system depicted in Fig. 12 is shown in Fig. 17. All other simulation parameters were the same as for the simulation result of Fig. 16. Such a relatively small misalignment angle leads to an output amplitude at the drive resonant frequency $f_{x}$, which is almost equal to the signal amplitude. Therefore, an effective correction mechanism should be adopted to avoid saturating the pickoff sensing amplifier. The quadrature force can be separated from the Coriolis signal force, since it is out of phase by $90^{\circ}$ with respect to the input signal.

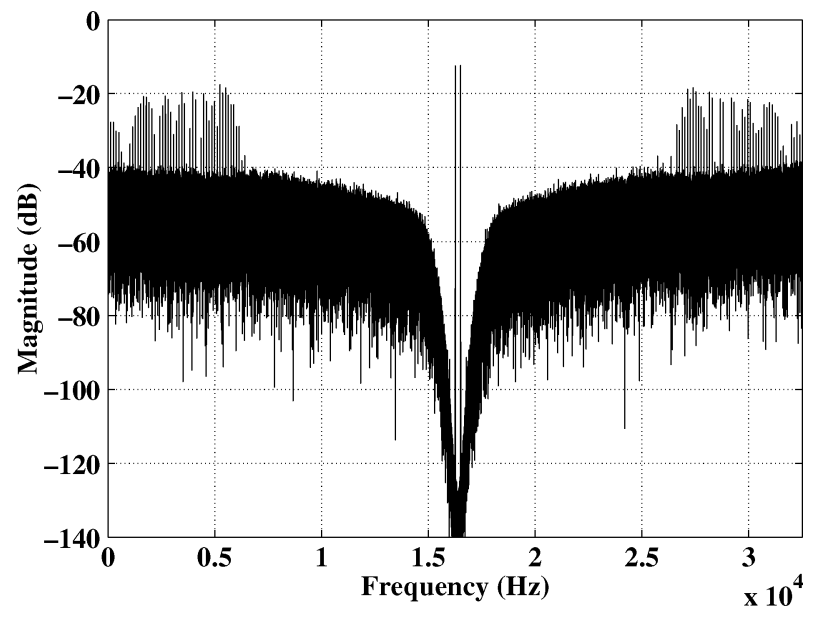

Fig. 16. Output bitstream spectrum of the eighth-order bandpass $\Sigma \Delta M$ with FFLRs (shown in Fig. 13).

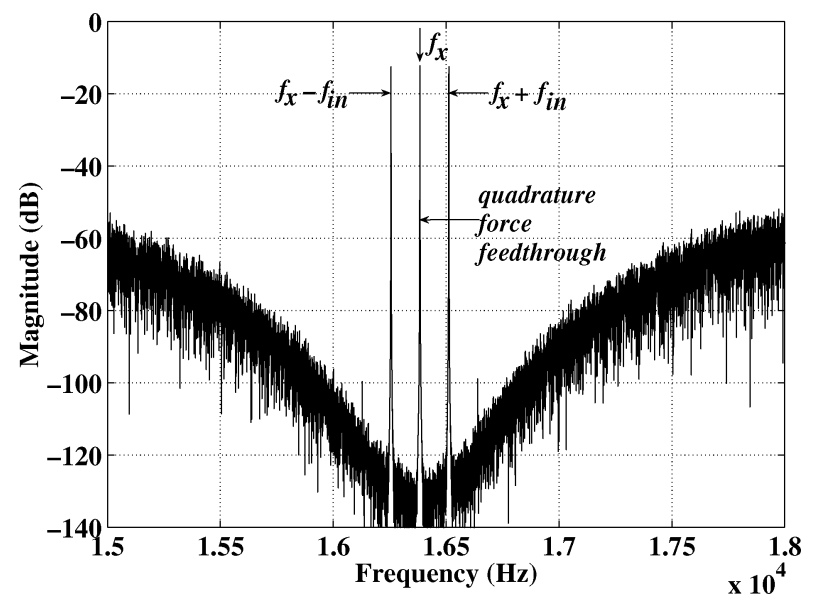

Fig. 17. Quadrature force effect on the output bitstream spectrum of the eighthorder bandpass $\Sigma \Delta \mathrm{M}$ with FFLRs (shown in Fig. 13).

\section{Resonant Frequency Mismatch Effect}

A resonant frequency mismatch between the drive mode and sense mode was not yet considered. Assuming a resonant frequency mismatch of $\pm 5 \%$ due to microfabrication tolerances, with all of the other simulation parameters the same, a simulation is done with the fifth-order low-pass $\Sigma \Delta \mathrm{M}$ (Fig. 3). The output bitstream spectrum of the system is shown in Fig. 18. There is a significant signal amplitude loss $(15 \mathrm{~dB})$ due to the mismatch compared with Fig. 5(b). While a simulation with the mismatch is done with the control system based on a bandpass $\Sigma \Delta \mathrm{M}$ (Fig. 13), the output spectrum (plotted in Fig. 19) shows that the spectrum is no longer perfectly symmetrically mirrored at $f_{x}$ and has a slightly higher noise floor compared with that of Fig. 16. Nevertheless, there is no signal amplitude degradation due to the resonant frequency mismatch, and this is a major advantage of a bandpass $\Sigma \Delta \mathrm{M}$ control loop.

\section{Effect of $1 / f$ Noise on the Control System Using a $\Sigma \Delta \mathrm{M}$}

The circuit implementation of a sensing and control system will inherently produce noise as stated above. Usually, CMOS analog circuits not only generate thermal noise, but also $1 / f$ noise. Lock-in or chopping modulation and CDS techniques are 


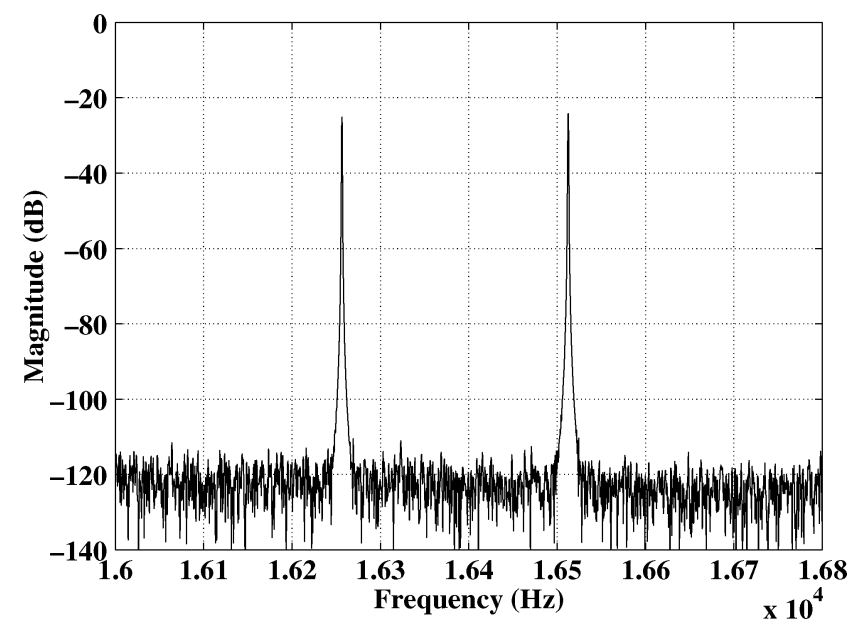

Fig. 18. Resonant frequency mismatch effect on the output bitstream spectrum of the fifth-order low-pass $\Sigma \Delta \mathrm{M}$ [compared with Fig. 5(b)].

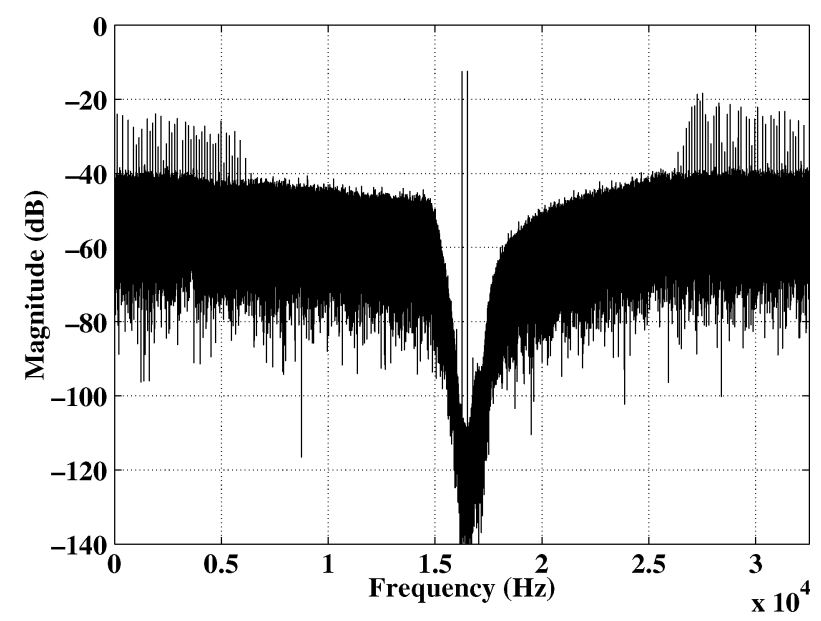

Fig. 19. Resonant frequency mismatch effect on the output bitstream spectrum of the eighth-order bandpass $\Sigma \Delta \mathrm{M}$ with FFLRs (shown in Fig. 13).

effective methods to remove $1 / f$ noise, drift, and offsets [17]. However, the $1 / f$ noise in any closed-loop gyroscope does not contribute to the overall electronic noise floor. This is due to the angular rate signal being synchronously modulated by the drive signal before it enters the preamplifier. This scheme shifts the $1 / f$ noise of the circuits out of the detection band. Therefore, the control systems based on both the low-pass and bandpass $\Sigma \Delta \mathrm{M}$ have good immunity to $1 / f$ and other low-frequency noise. The only potential problem for a low-pass control system is that it puts a relatively high calculation burden on the decimation stage of the digital signal processing due to its relatively high oversampling frequency.

\section{E. SNR Sensitivity to Fabrication Tolerances and Nonideal Circuit Implementation}

One of the key advantages of the control system using the $\Sigma \Delta \mathrm{M}$ approach is removing the need for precision analog circuits. Practical analog circuit implementations of an ideal electronic resonator, given by (10), are affected by the nonidealities of the operational amplifier, including finite gain, bandwidth, slew rate, and saturation voltages [16]. A Simulink model including these nonidealities is provided by [18] and shown in Fig. 20. The nonideal parameters used in this study are listed in

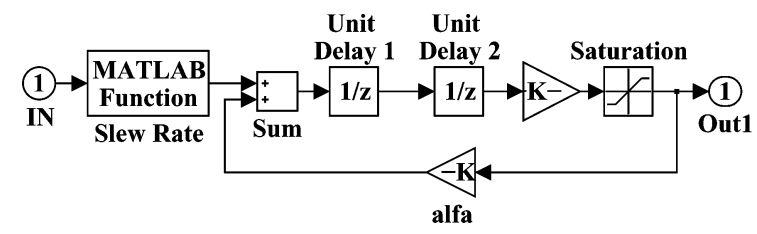

Fig. 20. Simulink model of a nonideal resonator [18].

TABLE II

NONIDEAL PARAMETERS OF AN OPERATIONAL AMPLIFIER

\begin{tabular}{c|c}
\hline Parameters & Values \\
\hline Open loop gain $A(\mathrm{~dB})$ & 60 \\
\hline Slew rate $[\mathrm{V} / \mu \mathrm{s}]$ & 1.0 \\
\hline GBW $[\mathrm{MHz}]$ & 1.0 \\
\hline Saturation value Amax $[\mathrm{V}]$ & \pm 1.2 \\
\hline
\end{tabular}

Table II, where alfa $=(A-1) / A$, and, for an ideal op-amp, alfa $=1$. All normal outputs of amplifiers are normalized to be $\pm 1 \mathrm{~V}$. The model was used for the simulation of the two high-order bandpass control systems. Due to the relatively low sampling frequency $(16 \mathrm{kHz})$, simulations show that there is a negligible performance degradation $(<1 \mathrm{~dB})$ due to these nonidealities. An operational amplifier with parameters given in Table II is easily implemented by modern CMOS technology.

It can be seen from (1) that the response of a micromachined sensing element $M_{m}(s)$ varies not only with frequency, but also with its mechanical parameters $\left[m, Q, \omega_{y}\right]$. The $S T F$ and $Q N T F$ of an electromechanical $\Sigma \Delta \mathrm{M}$ is not only sensitive to fabrication tolerances affecting the mechanical parameters, but also sensitive to variations of the electronic gain constants due to circuit nonidealities. The SNR may be degraded and, in the worst case, the loop may eventually become unstable. In this study, the SNR is used as the main criterion to measure the influence of fabrication tolerances on the performance of an electromechanical $\Sigma \Delta \mathrm{M}$. Another important criterion is the loop stability; this was assessed indirectly, as a sharp drop in SNR is a reliable indication for loop instability. In the following investigation, each parameter, which is subject to fabrication tolerances, is replaced by its nominal value plus or minus a random number generated with a uniform probability density function (a Matlab function unifrnd is used for the purpose). Relatively high worst case values are used here: for the nominal parameters of a sensing element ( $m_{0}=2 \mu \mathrm{g}, Q_{y}=100$, and $f_{y}=2^{14} \mathrm{~Hz}$ ), an uncertainty of $\pm 5 \%$ was assumed, while the electronic gain coefficient tolerance due to fabrication of the electronic circuits is comparatively small and was set to be $\pm 2 \%$ [19]. Monte Carlo simulations can then be used for parameter sensitivity analysis. In one simulation run, the input angular signal amplitude was varied from -60 to $40 \mathrm{~dB}$ (according to a full scale of $100^{\circ} / \mathrm{s}$ ). Each simulation for the criterion SNR was repeated 300 times. The SNR sensitivity to uncertainties of the values of the electronic gain stages was simulated first, and it was found that the SNR degraded from its ideal value by only $1 \mathrm{~dB}$. However, if mechanical fabrication tolerances were incorporated into the simulation, the effect is more significant, as shown in Fig. 21. The vertical length of each bar represents the SNR fluctuation which is approximately $16 \mathrm{~dB}$. It should be noted that the 


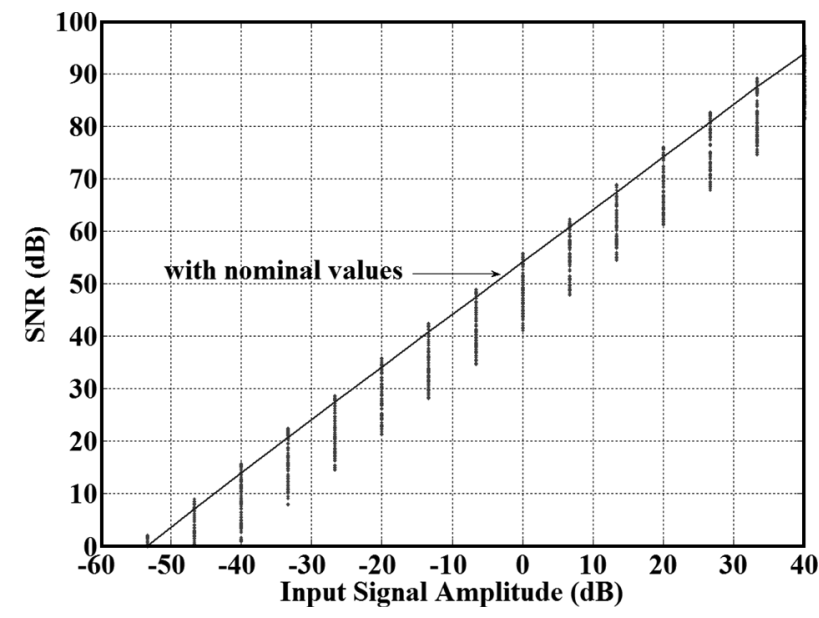

Fig. 21. Monte Carlo Simulation: SNR sensitivity to mechanical parameters and electronic stage gains.

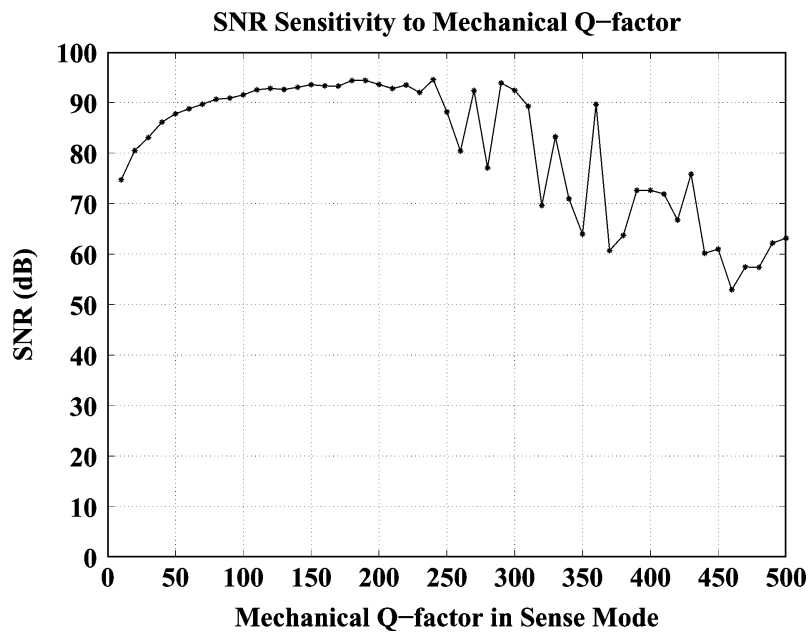

Fig. 22. SNR sensitivity to the mechanical quality $Q$ factor in the sense mode.

Monte Carlo simulations were carried out for the two high-order bandpass $\Sigma \Delta \mathrm{M}$ control systems (see Figs. 9 and 13), but the results were practically identical, and there is no effect on loop stability due to practical fabrication tolerances. The relatively large fluctuations of the SNR are mainly due to the compensator which is designed for nominal parameter values. Therefore, if the mechanical parameters $\left[m, Q_{y}, \omega_{y}\right]$ can be identified prior to designing the control system, the parameters of the compensator may be adjusted accordingly, and an ideal SNR can be achieved.

The parameter that is most affected by fabrication tolerances and changes in environmental conditions is usually the mechanical $Q$ factor of a vibratory gyroscope [20]. Therefore, it is worth investigating the sensitivity of the proposed control systems to the mechanical quality factor $Q$. For the high-order bandpass control system (shown in Fig. 9), the SNR sensitivity to the quality factor $Q_{y}$ in the sense mode is plotted in Fig. 22 with $Q$ ranging from 10 to 500 (its nominal value being 100). It can be seen that the SNR variation is relatively small for $Q_{y}$ ranging from 50 to 200. For lower $(<50)$ and higher $Q_{y}$ values $(>250)$, the control loop exhibits considerabl performance degradation. These results show that the control system is relatively insensitive to smaller variations in $Q_{y}$. However, if the quality factor changes significantly, the preamplifier gain of the control system needs to be reoptimized.

It should be noted that, to simplify the analysis in this paper, the mechanical Brownian noise has not been considered in the simulations because it directly adds to the input signal and is not shaped by any modulator topology.

\section{CONCLUSION}

This paper discusses a range of closed-loop force feedback control systems based on $\Sigma \Delta$ M's for the sense mode of vibratory-rate gyroscopes. It was found that a high-order bandpass $\Sigma \Delta \mathrm{M}$ topology, which cascades several electronic resonators with the micromachined sensing element, has distinct advantages over other topologies. Compared with a second-order $\Sigma \Delta \mathrm{M}$ loop, it provides much superior quantization noise shaping, making the quantization noise the nondominant noise source and furthermore alleviates idle tones. The advantages compared with a control system based on a high-order low-pass $\Sigma \Delta \mathrm{M}$ include a sampling frequency that is reduced by about two orders of magnitude and a wide and flat signal transfer function in the frequency range of interest. Two novel topologies of an eighth-order bandpass $\Sigma \Delta \mathrm{M}$ are discussed: a topology based on multifeedback loops with two local resonators and one based on feedforward loops with one local resonator. Both topologies achieve a SNR ratio of about $93 \mathrm{~dB}$ for typical parameter values for a micromachined sensing element and state-of-the art electronic components. The variations of mechanical parameters and electronic gain constants due to practical fabrication tolerances do not affect the loop stability, but the mechanical parameter tolerances have greater influence on system performance than the electronic gain tolerances.

\section{REFERENCES}

[1] N. Yazdi, F. Ayazi, and K. Najafi, "Micromachined inertial sensors," Proc. IEEE, vol. 86, no. 8, pp. 1640-1659, Aug. 1998.

[2] W. Henrion, L. D. Sanza, M. Ip, S. Terry, and H. Jerman, "Wide dynamic range direct digital accelerometer," in Proc. IEEE Solid State Sensor and Actuator Workshop, Hilton Head Island, SC, Jun. 1990, pp. 153-157.

[3] M. Lemkin and B. Boser, "A three-axis micromachined accelerometer with a CMOS position-sense interface and digital offset-trim electronics," IEEE J. Solid-State Circuits, vol. 34, no. 4, pp. 456-468, Apr. 1999.

[4] X. Jiang, J. Seeger, M. Kraft, and B. Boser, "A monolithic surface micromachined Z-axis gyroscope with digital output," in Proc. Symp. VLSI Circuits, Jun. 2000, pp. 16-19.

[5] V. Petkov and B. Boser, "A fourth-order $\Sigma \Delta$ interface for micromachined inertial sensors," IEEE J. Solid-State Circuits, vol. 40, no. 8, pp. 1602-1609, Aug. 2005.

[6] Y. Dong, M. Kraft, C. Gollasch, and W. Redman-White, "A high performance accelerometer with a fifth-order sigma-delta modulator," $J$. Micromech. Microeng., vol. 15, no. 7, pp. S22-S29, Jul. 2005.

[7] X. Jiang, "Capacitive position-sensing interface for micromachined inertial sensors," Ph.D. dissertation, Dept. Elect. Eng. and Comput. Sci., Univ. of California , Berkeley, 2003.

[8] S. Norsworth, R. Schreier, and C. Temes, Delta-Sigma Data Converters: Theory, Design, and Simulation. New York: IEEE Press, 1997.

[9] Y. Dong, M. Kraft, and W. Redman-White, "High order bandpass sigma delta interfaces for vibratory gyroscopes," in Proc. 4th IEEE Conf. Sensors, Irvine, CA, 2005, pp. 1080-1083.

[10] Z.-M. Lin and W.-H. Sheu, "A generic multiple-feedback architecture and method for the design of high-order $\sigma \delta$ modulators," IEEE Trans. Circuits Syst. II, Analog Digit. Signal Process., vol. 49, no. 7, pp. 465-473, Jul. 2002. 
[11] Y. Dong, "Control systems using high order Sigma Delta modulators for capacitive micromachined inertial sensors," Ph.D. dissertation, School of Electron. Comput. Sci., Univ. of Southampton, Southampton, U.K., Jun. 2006.

[12] M. Neitola, A Fully Automated Flowgraph Analysis Tool for MATLAB 2006 [Online]. Available: http://www.mathworks.com/matlabcentral/fileexchange/loadFile.do?objectI\% d=7224\&objectType $=$ file

[13] C. Acar and A. M. Shkel, "An approach for increasing drive-mode bandwidth of MEMS vibratory gyroscopes," IEEE/ASME J. Microelectromech. Syst., vol. 14, no. 3, pp. 520-528, Mar. 2005.

[14] G. Franklin, J. Powell, and A. Emami-Naeini, Feedback Control of Dynamic Systems. Upper Saddle River, NJ: Prentice Hall, 2005.

[15] P. Cusinato, F. Pasolini, F. Stefani, and A. Baschirotto, "Digital technique for instability detection and saturation recovery in high-order SC $\Sigma \Delta$ modulators," Analog Integr. Circuits Signal Process., vol. 36, no. 1-2, pp. 7-19, Jul./Aug. 2003.

[16] S. Brigati, F. Francesconi, P. Malcovati, and F. Maloberti, "A fourth-order single-bit switched-capacitor $\Sigma \Delta$ modulator for distributed sensor applications," IEEE Trans. Instrum. Meas., vol. 53, no. 2, pp. 266-270, Apr. 2004.

[17] C. Enz and G. Temes, "Circuit techniques for reducing the effects of op-amp imperfections: Autozeroing, correlated double sampling, and chopper stabilization," Proc. IEEE, vol. 84, no. 11, pp. 1584-1614, Nov. 1996.

[18] P. Malcovati, SD Toolbox 2, MatLab File Exchange 2005 [Online]. Available: http://www.mathworks.com/matlabcentral/fileexchange/loadFile.do?objectI\% d=7589\&objectType=file

[19] T.-H. Kuo, K.-D. Chen, and J.-R. Chen, "Automatic coefficients design for high-order sigma-delta modulators," IEEE Trans. Circuits Syst. II, Analog Digit. Signal Process., vol. 46, no. 1, pp. 6-15, Jan. 1999.

[20] C. Jeong, S. Seok, B. Lee, H. Kim, and K. Chun, "A study on resonant frequency and Q factor tunings for MEMS vibratory gyroscopes," $J$. Micromech. Microeng., vol. 14, no. 11, pp. 1530-1536, 2004.

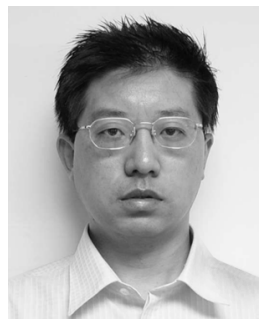

Yufeng Dong received the M.S. degree in automation from Tsinghua University, Beijing, China, in 1993, and the Ph.D. degree in electrical engineering from the University of Southampton, Southampton, U.K., in 2006.

In March 1993, he joined Tsinghua University as a Lecturer, where he was involved with the research of high-resolution X-ray industrial tomography for ceramic engines and 3-D micro-tomography, which were supported by the 863 National High-Tech Research and Development Plan of China and the National Natural Science Foundation of China, respectively. In November 1997, he joined GE Medical Systems as a Senior Staff Design Engineer, developing data acquisition systems and X-ray solid-state detectors for high-speed CT systems. Since October 1999, he has been the CTO of the start-up Wangtong Technologies, Beijing, China, where he was in charge of the EDA consulting services and SoC hardware validation. He is currently a Postdoctoral Research Fellow with the School of Electronics and Computer Science, University of Southampton, Southampton, U.K., working in the area of analog and mixed-signal integrated circuits, with special emphasis on MEMS sensors and control systems.

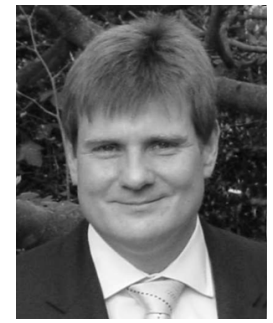

Michael Kraft received the Dipl.-Ing. (Univ.) degree in electrical and electronics engineering from the Friedrich Alexander Universitat, Erlangen-Nürnberg, Germany, in 1993, and the Ph.D. degree from Coventry University, Coventry, U.K., in 1997.

In the same year, he joined the Nonlinear System Design Group, Coventry University, where he worked on the design of a digital micromachined accelerometer. He then spent two years at the Berkeley Sensors and Actuator Center, University of California, Berkeley, working on the design of integrated micromachined gyroscopes. He is currently a Senior Lecturer with the School of Electronics and Computer Science, University of Southampton, Southampton, U.K. His principle interests include micromachined inertial sensors, MEMS sensors and actuators, intelligent control systems for MEMS devices, and electronic circuit design.

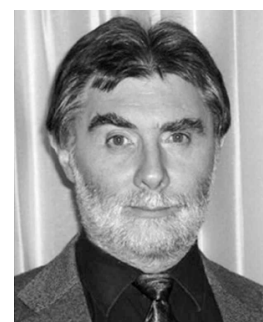

William Redman-White (M'83) received the M.Sc. and Ph.D. degrees from Southampton University, Southampton, U.K., in 1980 and 1983, respectively.

He has been with Philips Semiconductors since 1990, working in Southampton, U.K., San Jose, CA, and Caen, France, on optical storage, WLAN, cellular radio, Bluetooth, digital audio, TV and satellite baseband, presently as a Fellow in Southampton, U.K. He has previously been with Motorola, Geneva, GEC-Marconi Research London, and Post Office Telecommunications, London. Concurrently with his industrial activities, he has also had a faculty position in Southampton University since 1983, currently as a full Professor of integrated circuit design. His research and teaching is centered on analog and RFIC design, and design issues in SOI CMOS technology. He has published over 100 papers and has had more than 10 patents granted with several pending.

Dr. Redman-White served as an Associate Editor for the IEEE JOURNAL OF Solid-STATE CIRCUITS from 1996 to 2002, is a member of the IEEE International Solid-State Circuits Conference committee, and is part of the steering committee for the European Solid State Circuits and Devices Conferences (ESSCIRC/ESSDERC) 